\title{
Is there a role for Amniotic Membrane as an antifibrotic agent in MMC-Augmented Trabeculectomy?
}

Joana Roque ( $\sim$ joananunesroque@gmail.com )

Hospital Professor Doutor Fernando Fonseca EPE https://orcid.org/0000-0002-0713-7064

Fernando Trancoso Vaz

Hospital Professor Doutor Fernando Fonseca EPE

Rita Basto

Hospital Professor Doutor Fernando Fonseca EPE

Susana Henriques

Hospital Professor Doutor Fernando Fonseca EPE

Ana Sofia Lopes

Hospital Professor Doutor Fernando Fonseca EPE

Diana Silva

Hospital Professor Doutor Fernando Fonseca EPE

Jorge Santos

IIFA: Universidade de Evora Instituto de Investigacao e Formacao Avancada

Graça Pires

Hospital Professor Doutor Fernando Fonseca EPE

Maria Lisboa

Hospital Professor Doutor Fernando Fonseca EPE

Isabel Prieto

Hospital Professor Doutor Fernando Fonseca EPE

\section{Research Article}

Keywords: trabeculectomy, glaucoma, mitomycin C, amniotic membrane, antifibrotics

Posted Date: March 9th, 2021

DOl: https://doi.org/10.21203/rs.3.rs-178110/v1

License: (c) (i) This work is licensed under a Creative Commons Attribution 4.0 International License.

Read Full License 


\section{Is there a role for Amniotic Membrane as an antifibrotic agent in MMC-Augmented Trabeculectomy?}

Roque $\mathrm{J}^{1}$; Vaz Fernando T ${ }^{1}$; Basto R ${ }^{1}$; Henriques $\mathrm{S}^{1}$; Lopes $\mathrm{A}^{1}$; Silva $\mathrm{D}^{1}$; Santos $\mathrm{J}^{2}$; Pires $\mathrm{G}^{1}$; Lisboa $\mathrm{M}^{1}$; Prieto $\mathrm{I}^{1}$ ${ }^{1}$ Ophthalmology Department, Prof. Doutor Fernando Fonseca Hospital, Amadora, Lisbon, Portugal

${ }^{2}$ MathDep. ECT, CIMA IIFA Évora University, Évora, Portugal

Corresponding author: Joana Roque, MD (ORCID identifier 0000-0002-0713-7064). Correspondance: Ophthalmology Department, Prof. Doutor Fernando Fonseca Hospital, IC19 2720-276 Amadora, Portugal. E-mail: joana.roque@hff.minsaude.pt Telephone number: +351932828099 .

\section{Declarations}

\section{Data Availability}

Investigational data is available if requested.

\section{Animal Research (Ethics)}

Not applicable.

\section{Consent to Participate (Ethics)}

All patients signed the requested informed consent.

\section{Consent to Publish (Ethics)}

All patients signed the requested informed consent to publish this study. Consent to publish was also conceded by the Hospital Ethics Committee.

\section{Clinical Trials Registration}

Not applicable.

\section{Author Contribution}

All authors contributed to the study conception and design. Material preparation, data collection and analysis were performed by Joana Roque, Fernando Vaz, Rita Basto, Susana Henriques, Ana Lopes and Diana. Statistical analysis was performed by Jorge Santos. The first draft of the manuscript was written by Joana Roque. Fernando Vaz, Graça Pires, Maria Lisboa and Isabel Prieto proofread the manuscript and figure legends, and provided important guidance and oversight during the entire drafting process All authors read and approved the final manuscript.

\section{Conflict of Interest}

The authors declare that they have no conflict of interest.

\section{Funding}

This study did not receive any sum from funding agencies in the public, commercial or not-for-profit sectors 


\section{Abstract}

Purpose: Amniotic membrane transplantation (AMT) has shown promising results as an antifibrotic agent in trabeculectomy. We aimed to evaluate the additional effect of AMT in MMC-augmented trabeculectomy.

Methods: This retrospective study analyzed the results of the first 12 postoperative months of glaucomatous eyes submitted to Moorfields Safer Surgery Trabeculectomy with MMC alone (non-AMT group) compared to MMC and AMT (AMT group). Both groups were compared in terms of intraocular pressure (IOP), number of antihypertensive medications and need for surgical reinterventions. Absolute and relative success rates 12 months after surgery were defined as IOP $<18 \mathrm{mmHg}$, without and with the use of antihypertensive medications, respectively.

Results: The analysis included 51 eyes of 45 glaucoma patients (29 eyes in the non-AMT group and 22 in the AMT group). Mean IOP decreased from 24,72 $\pm 5,11 \mathrm{mmHg}$ and $26,86 \pm 10,62 \mathrm{mmHg}$ preoperatively in non-AMT and AMT groups to $12,86 \pm 4,22 \mathrm{mmHg}$ and $12,60 \pm 4,43 \mathrm{mmHg}$, respectively, at 12 months $(\mathrm{p}=0,84)$. Postoperative number of medications decreased significantly in both groups. Absolute success was seen in $71 \%$ of non-AMT eyes and $55 \%$ of AMT eyes $(p=0,46)$ whereas relative success was obtained in $14 \%$ and $30 \%$, respectively $(p=0,55)$. Reinterventions were needed in $28 \%$ of the eyes (11 bleb injection/needling and 4 Ahmed tube implantation) in the non-AMT group and in $27 \%$ of the AMT group (10 bleb injection/needling and 1 Ahmed tube implantation) $(\mathrm{p}=0,89)$.

Conclusion: Trabeculectomy combined with MMC and AMT did not show better results than trabeculectomy with MMC alone.

Keywords: trabeculectomy, glaucoma, mitomycin C, amniotic membrane, antifibrotics 


\section{Introduction}

Trabeculectomy is the gold standard procedure in glaucoma surgery worldwide[1]. It creates a drainage pathway between the anterior chamber and the subconjunctival space leading to a subconjunctival reservoir for the aqueous humor, referred to as the filtering bleb.

The technique was first described in 1968 by Cairns [2]. Since then several modifications to this procedure, such as the 'Moorfields Safer Surgery System' (MSS Trab) [3] have improved surgical safety, predictability and outcomes. Current procedures aim to achieve larger and more diffuse filtering blebs, less prone to long-term failure, leakage and infectious complications.

Nevertheless the wound healing process that occurs after surgery still leads to an excessive fibrosis at the subconjunctival space. This represents the ultimate reason for bleb failure and uncontrolled IOP after surgery. Therefore glaucoma surgeons use antifibrotic agents such as Mitomycin C (MMC) and 5-Fluorouracil (5-FU) in a great proportion of the eyes undergoing trabeculectomy, according to the individuals' risk of bleb failure[3]. None of these antifibrotic agents has however completely superseded conjunctival scarring. On the other hand, theses agents are associated with several short and long-term complications, such as bleb avascularity, thinning of the conjunctiva, hypotony, and increased risk of endophthalmitis[4], hence the need for more specific modulators of the wound-healing pathway.

Newer adjunctive agents have been investigated in order to increase bleb survival. These include anti-vascular endothelial growth factor (VEGF) agents[5, 6], space occupying components such as the Ologen ${ }^{\circledR}$ implant[7], and also amniotic membrane transplantation (AMT). Although none of these has clearly supplanted MMC-augmented trabeculectomy, amniotic membrane has been increasingly investigated in glaucoma surgery, since it was first described in 1998[8]. Human amniotic membrane is widely known for its anti-inflammatory, antifibrotic and antiangiogenic properties, distinctly acting at different levels of the wound healing process. It has shown promising results as a physiological bleb modulator in glaucoma filtering surgeries[9-11].

There is some data suggesting a favorable effect of the combination of AMT with MMC-augmented trabeculectomy [1214] but it is still unclear if this should be a routine procedure. This study evaluated the results of glaucoma patients submitted to trabeculectomy with MMC compared to patients submitted to trabeculectomy with simultaneous use of MMC and amniotic membrane.

\section{Methods}

This retrospective comparative study analyzed the results of the first 12 postoperative months of glaucoma patients submitted to trabeculectomy at the Glaucoma Department of Prof. Doutor Fernando Fonseca Hospital, Amadora, Lisbon, Portugal, from January 2015 to May 2018. The study respected the tenets of the Declaration of Helsinki and received the approval of the Hospital Ethics Committee.

\section{Patients and treatments}

Clinical data from 51 eyes of 45 glaucoma patients (open-angle and angle-closure glaucoma) were retrospectively analyzed. Among them, 29 eyes were treated with MMC-augmented trabeculectomy (non-AMT group) and 22 eyes were treated with MMC-augmented trabeculectomy combined with amniotic membrane transplantation (AMT group). The latter were operated mainly since the beginning of 2017, once AMT was a more established procedure. We did not include data from patients who had had glaucoma surgery before nor patients whose clinical records were incomplete.

\section{Surgical methods}

All trabeculectomies were performed by the same two surgeons according to Moorfields Safer Surgery System[3]. A fornix-based conjunctival flap was made on the upper margin of the temporal quadrant, along with an extended and careful subconjunctival dissection. A scleral flap of $4 \times 4 \mathrm{~mm}$ with $1 / 3$ thickness of sclera was cut $0,5 \mathrm{~mm}$ from the limbus. Polyvinyl alcohol sponges soaked in MMC $(0,2 \mathrm{mg} / \mathrm{mL}$ or $0,4 \mathrm{mg} / \mathrm{mL}$ ) were applied in the largest possible area of the subconjunctival space and under the scleral flap for 3 minutes, followed by a thorough washing with saline

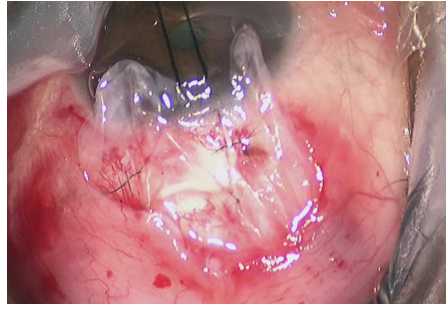

Fig. 1- Amniotic membrane above the scleral flap (stromal surface facing down). 
solution. MMC concentration was selected according to Moorfields/Florida 'More Flow' regime[3], which stratifies patients into three levels of risk for trabeculectomy failure. Afterwards, using an anterior chamber maintainer, a standardized sclerostomy was performed with a $0,5 \mathrm{~mm}$ punch. After iridectomy, the scleral flap was sutured with two fixed sutures along with two adjustable/removable sutures. For patients in the AMT group, before suturing the conjunctiva, the previously defrosted human amniotic membrane was implanted above the scleral flap (Fig. 1) with the stromal surface facing upwards (allowing the antifibrotic mediators to contact with the subconjunctival fibroblasts). The single-layered amniotic membrane was then fixed near the limbus with 2 sutures (10/0 nylon) and spread towards the fornix. The conjunctiva was sutured with two interrupted $6 / 0$ Vycril sutures at the ends of the conjunctival incision Postoperative management included application of topical dexamethasone phosphate $0,1 \% \mathrm{q} 4 \mathrm{~h}$ and ofloxacin $0,3 \% \mathrm{q} 6 \mathrm{~h}$, tapered over several weeks.

\section{Postoperative follow-up and efficacy evaluation}

Pre and postoperative IOP, number of antihypertensive medications, as well as need for surgical reinterventions (5-FU or MMC bleb injection/needling or glaucoma drainage device implantation) data were collected from the following follow-up visits: week 1 and months 1, 2, 3, 4, 5, 6, 8 and 12 .

Filtering bleb morphology/quality is a subjective characteristic difficult to assess retrospectively (large and diffuse, flat, cystic, avascular, etc.) (Fig. 2). Thus to evaluate the procedures' efficacy, we defined success criteria and analyzed the need and the timing for reinterventions in those eyes presenting with nonfunctioning blebs. Absolute success was defined as IOP $<18 \mathrm{mmHg}$ without antihypertensive medications 12 months after operation, and relative success as IOP $<18 \mathrm{mmHg}$ with the use of IOP-lowering medications at the same timepoint.

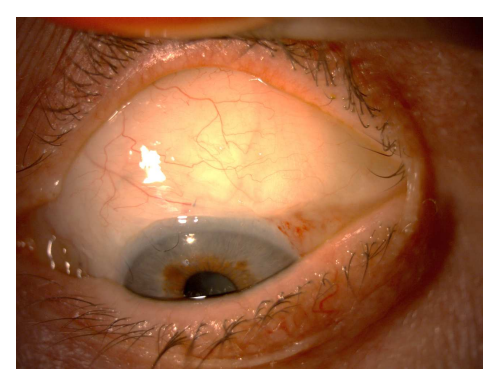

Fig. 2- Large and diffuse filtration bleb registered in one of the eyes of the AMT group.

\section{Statistical analysis}

The statistical analysis was performed by a statistian, using IBM ${ }^{\circledR}$ SPSS ${ }^{\circledR}$ Statistics. Continuous variables were analyzed using the independent Student's t-test and Welch's unequal variances t-test. The categorical variables were analyzed using the chi-square test or Fisher's exact test depending on sample size. A p<0,05 was considered significantly different.

\section{Results}

\section{Preoperative Baseline Characteristics}

Fifty-one glaucomatous eyes were analyzed in this study (29 eyes in the non-AMT group and 22 eyes in the AMT group).

As shown in Table 1, preoperative baseline characteristics were similar between both groups. Overall mean preoperative IOP (95\% confidence interval) was $25,72 \pm 2,39 \mathrm{mmHg}$.

Table 1- Preoperative baseline characteristics

\begin{tabular}{|l|l|l|l|}
\hline Parameter & $\begin{array}{l}\text { Non-AMT group } \\
\mathbf{n = 2 9}\end{array}$ & $\begin{array}{l}\text { AMT group } \\
\mathbf{n = 2 2}\end{array}$ & p-value \\
\hline Mean preoperative IOP \pm SD & $24,72 \pm 5,11 \mathrm{mmHg}$ & $26,86 \pm 10,62 \mathrm{mmHg}$ & $0,40^{\mathrm{a}}$ \\
\hline $\begin{array}{l}\text { Age } \\
\text { Mean }\end{array}$ & $\begin{array}{l}65 \text { years } \\
\text { Range }\end{array}$ & $\begin{array}{l}64 \text { years } \\
29-78 \text { years }\end{array}$ & $0,72^{\mathrm{b}}$ \\
\hline Gender (F:M) & $16: 13$ & $6: 16$ & $0,08^{\mathrm{c}}$ \\
\hline
\end{tabular}

Abbreviations: SD- Standard Deviation; F- Female; M- Male; a- Welch's unequal variances t-test; b- Student's t-test; c- Fisher's exact test.

The study included eyes with both open angle and angle closure glaucoma: 23 eyes with primary open angle glaucoma (POAG), 14 eyes with primary angle closure glaucoma, 10 eyes with pseudoexfoliative glaucoma, 3 eyes with juvenile glaucoma and 1 eye with steroid-induced glaucoma. 


\section{Postoperative IOP, Medications and Success Rates}

Follow-up time considered for the study was a minimum of 12 months for all patients. Mean postoperative IOP values in both groups are presented in Table 2. Figure 3 shows mean IOP evolution in both groups during the twelve-month period. Postoperative mean IOP in the AMT group was significantly lower $(\mathrm{p}=0,02)$ than non-AMT group only at the first postoperative week (7,05 and 10,37 $\mathrm{mmHg}$, respectively). On follow-up months $1,3,6$, and 12, mean IOP was lower in the AMT group however without statistical significance.

Table 2- Postoperative mean IOP and standard deviations at follow-up visits

\begin{tabular}{|l|l|l|l|}
\hline & Non-AMT group $(\mathbf{m m H g})$ & AMT group $\mathbf{( m m H g )}$ & p-value \\
\hline 1 week & $10,37 \pm 5,47$ & $7,05 \pm 2,13$ & 0,02 \\
\hline 1 month & $13,87 \pm 6,12$ & $12,57 \pm 7,35$ & 0,53 \\
\hline 3 months & $14,73 \pm 5,55$ & $14,33 \pm 4,79$ & 0,81 \\
\hline 6 months & $13,58 \pm 3,85$ & $12,07 \pm 3,91$ & 0,28 \\
\hline 12 months & $12,86 \pm 4,22$ & $12,60 \pm 4,43$ & 0,84 \\
\hline
\end{tabular}

Fig. 3- Preoperative and postoperative mean IOP during follow-up period

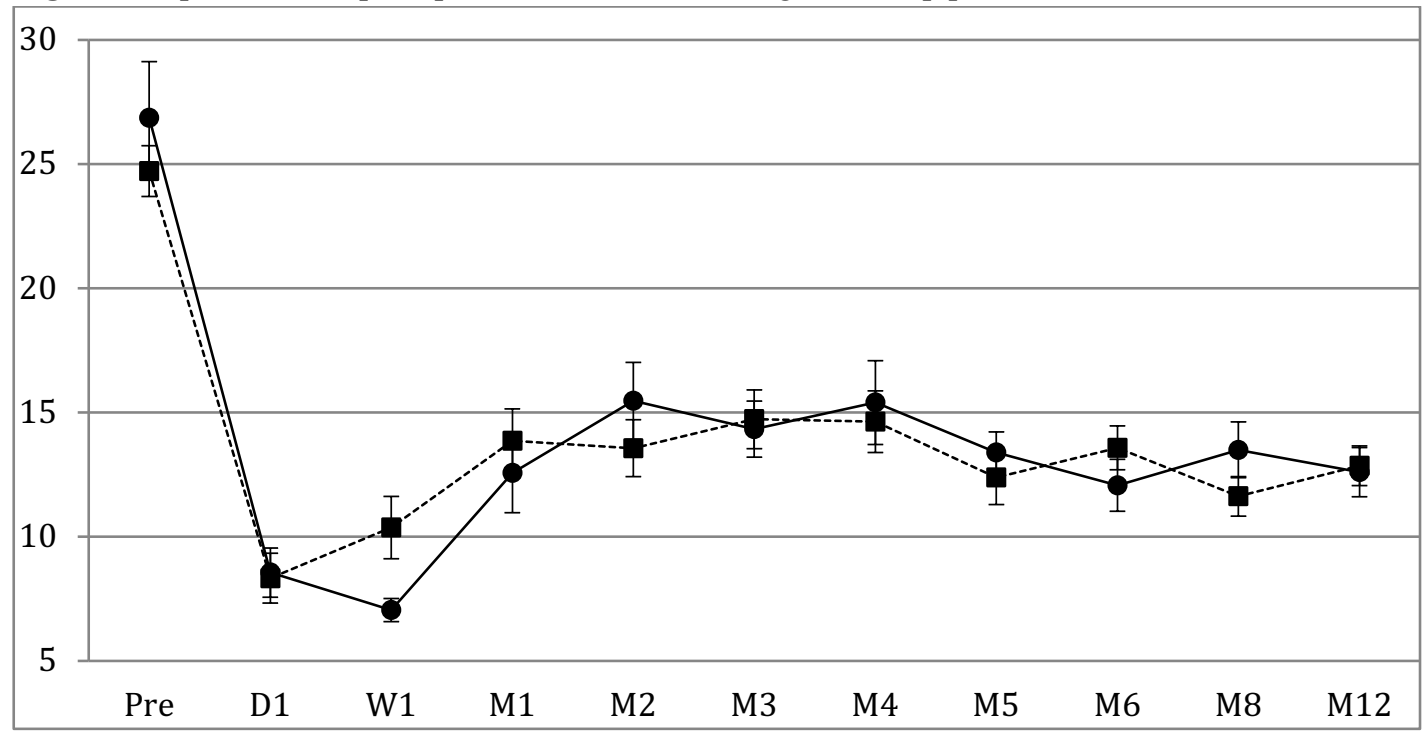

We present mean IOP values in each study group. The bars represent the standard error at each follow-up visit.

Vertical axis- Mean IOP in each group; Horizontal axis- Preoperative and postoperative visits; Solid line- non-AMT group; Dashed line- AMT group.

Patients in both study groups used a mean of 4 hypotensive topical medications preoperatively, which decreased significantly in both groups. Along the studied period the number of IOP-lowering medications tended to be higher in the AMT group, with a significant difference at 6 months after surgery $(1,83$ vs 0,32$)$ (Fig. 4). Twelve months after surgery, mean number of medications was 0,61 in the non-AMT group and 1,4 in the AMT group $(\mathrm{p}=0,06)$. 
Fig. 4- Preoperative and postoperative mean number of antihypertensive medications during follow-up period

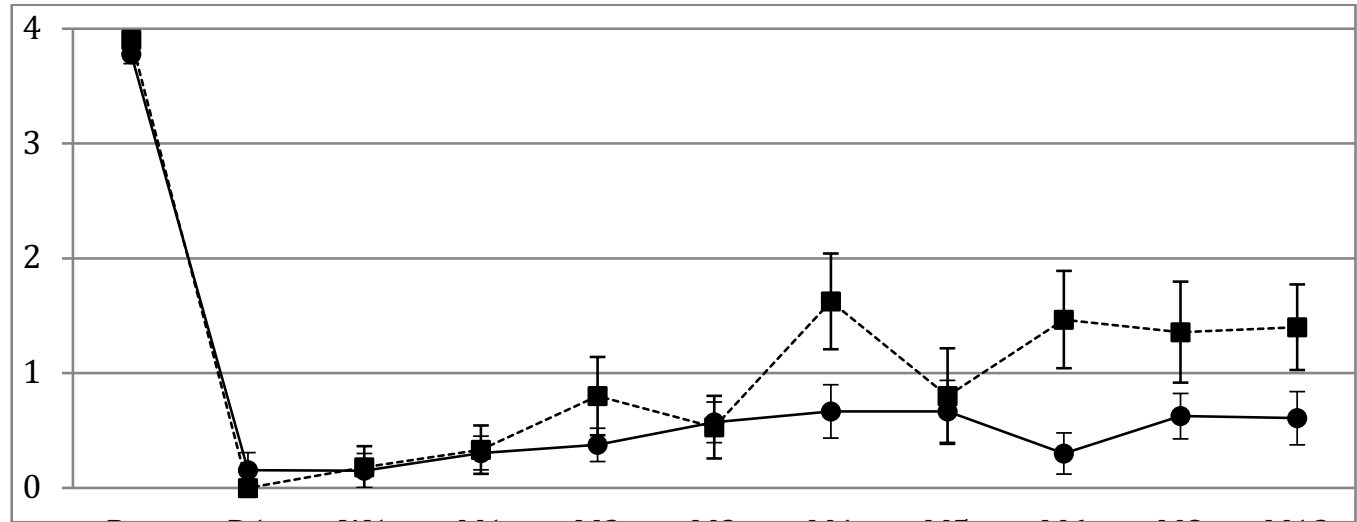

Representation of the mean number of antihypertensive medications used in each group, along with bars relative to the standard error at each timepoint. Vertical axis- Mean number of antihypertensive medications in each group; Horizontal axis- Preoperative and postoperative visits; Solid line- non-AMT group; Dashed line- AMT group.

Regarding success rates, absolute success was higher in the non-AMT group than in the AMT group, 71\% and 55\% respectively $(\mathrm{p}=0,46)$. Relative success rates were accomplished in $14 \%$ and in $30 \%$ of the eyes in the non-AMT and AMT groups, respectively ( $\mathrm{p}=0,55)$. Disregarding the use of hypotensive medications, $86 \%$ of the eyes in the non-AMT group and $85 \%$ of the eyes in the AMT groups achieved IOP below the considered cutoff $(\mathrm{p}=0,63)$.

\section{Need for Reinterventions}

Surgical reinterventions were needed in 8 eyes $(28 \%)$ of the non-AMT group and in 6 eyes $(27 \%)$ of the AMT group (p= 0,89 ). Table 3 specifies the type of procedures performed in each group. The eyes in the non-AMT group required 15 reinterventions: 11 5-FU bleb injections/needling and 4 surgeries for Ahmed tube implantation. The ones in the AMT group were submitted to 11 reinterventions: 3 5-FU bleb injections/needling, 7 MMC bleb injections/needling and 1 Ahmed tube implantation. We did not find statistical significance in the number of eyes that showed complete failure of trabeculectomy culminating in glaucoma drainage device (GDD) implantation (4/29 eyes in non-AMT group and $1 / 22$ eyes in AMT group, $\mathrm{p}=0,27)$. Mean time until first reintervention was also similar: 3,1 months in the non-AMT group and 2 months in the AMT group.

Table 3- Type of reintervention performed

\begin{tabular}{|l|l|l|}
\hline Type of reintervention & $\begin{array}{l}\text { Non-AMT group } \\
\mathbf{n = 2 9}\end{array}$ & $\begin{array}{l}\text { AMT group } \\
\mathbf{n}=\mathbf{2 2}\end{array}$ \\
\hline 5-FU bleb injection/needling & $11(7$ eyes $)$ & 3 ( 2 eyes $)$ \\
\hline MMC bleb injection/needling & None & 7 (5 eyes $)$ \\
\hline GDD implantation (Ahmed tube) & 4 (4 eyes) & 1 ( 1 eye $)$ \\
\hline Total & $15(8$ eyes $)$ & $11(6$ eyes $)$ \\
\hline
\end{tabular}

Abbreviations: GDD- Glaucoma drainage device.

Note: Some of the eyes subject to bleb injection/needling were subsequently submitted to a GDD surgery.

\section{Discussion}

The healing process at the filtration bleb is the main reason for trabeculectomy failure, thus wound healing modulation has been thoroughly investigated as the key element to improve trabeculectomy outcomes.

Human amniotic membrane is the innermost layer of the placenta. It serves as a valuable adjunct tool for ocular surgery because of its many beneficial features: it is transparent, lacks immunogenicity, and has been shown to have antiinflammatory, antifibrotic, antiangiogenic, and possibly antimicrobial properties. It is primarily used in the treatment of ocular surface disorders, serving as a substrate for epithelium to grow on. On the other hand, the stromal surface of amniotic membrane stands out for downregulating Tumor Growth Factor- $\beta$ signaling, thus reducing fibroblast production 
and myofibroblast differentiation, leading to decreased scar formation. It also inhibits proinflammatory cytokines and inflammatory cells migration, resulting in a reduced inflammatory reaction and vascularization[15].

Based on these properties, amniotic membrane has been proposed as an adjunct to trabeculectomy, in order to prevent healing in the subconjunctival space and to promote long-living blebs. Experimental rabbit studies have demonstrated that amniotic grafts at the trabeculectomy site lead to less fibroblast cell counts and better bleb function[16, 17]. Since Fujishima's study in 1998[8], several clinical studies pointed to the favorable outcomes of addition of AMT to trabeculectomy without antifibrotics[9], however some of them lacking statistical significance. The randomized trial performed by Eliezer et al. [18] did not find a statistically significant IOP-lowering effect of a folded amniotic membrane graft in classic trabeculectomy. More recently, Stavrakas and his colleagues[11] enrolled 59 eyes with POAG in a prospective randomized trial over 24 months comparing trabeculectomy without antimetabolites to trabeculectomy combined with AMT. The reduction of IOP was greater in the AMT group throughout the follow-up period but this difference was not statistically significant at any point.

Other authors compared trabeculectomy with AMT versus trabeculectomy with antifibrotics. The randomized trial conducted by Khairy et al.[10], involving 52 eyes with POAG, revealed that trabeculectomy with AMT had a comparable IOP-lowering effect but a reduced rate of postoperative complications after 24 months of follow-up compared to trabeculectomy with MMC.

To the best of our knowledge, only three studies have compared the simultaneous use of MMC and AMT in trabeculectomy. In 2008, Sheha and colleagues [12] performed a randomized trial in 37 eyes with refractory glaucoma, comparing trabeculectomy with MMC and single-layer AMT under the scleral flap and trabeculectomy with MMC alone. Twelve months after surgery, results showed that AMT was associated with higher success rates, lower postoperative mean IOP and less complication rates in that group of patients. In 2010, Mahdy and others[14] studied 30 eyes with pediatric glaucoma divided into two groups: trabeculectomy with MMC and AMT, and trabeculectomy with MMC alone. After 18 months of follow-up, there was no significant difference in the IOP-lowering effect but the group with AMT showed fewer complications. In 2017, Yadava et al. [13] randomized 40 eyes with uncontrolled glaucoma (37 of them with PACG) into two groups: control group underwent trabeculectomy augmented with MMC while the study group underwent additional AMT. At one-year follow-up, the latter achieved higher rates of complete success in IOP reduction and lesser interventions such as bleb needling.

We conducted this retrospective comparative study in order to further analyze the simultaneous use of amniotic membrane and MMC. Our study is the first comparative study using MSS Trab. Overall the good results in terms of IOP control and complications are according to the outcomes of this type of surgery.

However the results did not prove a favorable effect of simultaneous use of MMC and AMT. We did not find significantly different postoperative IOP values, and surprisingly there was a higher need for antihypertensive medications in the AMT group. The need for surgical reinterventions reflected the existence of nonfunctioning blebs, whether they were flat or cystic. In the twelve-month period, the percentage of eyes that required surgical reinterventions was similar in both groups, as well as the timing of those procedures.

The aforementioned results could be limited by the retrospective nature of the investigation, by the unequal number of eyes in both study groups and by the heterogeneity of glaucoma types among them. We must point the possible bias of the higher number of antihypertensive medications used in the AMT group. However tendentiously higher levels of IOP encountered in this group could explain this difference. Besides, the authors performed AMT using one single layer of amniotic membrane in contrast to several previous reports of folded or double layer grafts, which could eventually lead to different outcomes.

Search for new alternatives for fibrosis control is still a challenge in glaucoma surgery. Amniotic membrane could be further studied, whether used as a folded graft or in combination with other antifibrotic adjuncts. 


\section{Conclusion}

Despite the known antifibrotic properties of amniotic membrane, in our study its addition to MMC-augmented trabeculectomy did not improve surgical outcomes in terms of IOP and need for medications or surgical reinterventions. Further investigations with prospective clinical trials are needed to better understand the role of amniotic membrane in glaucoma surgery. On the other hand, the search for new alternatives to antimitotic drugs is still imperative.

\section{References}

1. Higginbotham EJ, Alexis D. Is Newer Necessarily Better? The Evolution of Incisional Glaucoma Surgery Over the Last 100 Years. Am J Ophthalmol. 2018;191:xxv-xxix. doi: 10.1016/j.ajo.2018.04.009.

2. Cairns JE. Trabeculectomy. Preliminary report of a new method. Am J Ophthalmol. 1968;66(4):673-9.

3. Khaw PT, Chiang M, Shah P, Sii F, Lockwood A, Khalili A. Enhanced Trabeculectomy: The Moorfields Safer Surgery System. Dev Ophthalmol. 2017;59:15-35. doi: 10.1159/000458483.

4. Yoon PS, Singh K. Update on antifibrotic use in glaucoma surgery, including use in trabeculectomy and glaucoma drainage implants and combined cataract and glaucoma surgery. Curr Opin Ophthalmol. 2004;15(2):141-6.

5. Slabaugh M, Salim S. Use of Anti-VEGF Agents in Glaucoma Surgery. J Ophthalmol. 2017;2017:1645269. doi: $10.1155 / 2017 / 1645269$.

6. Akkan JU, Cilsim S. Role of subconjunctival bevacizumab as an adjuvant to primary trabeculectomy: a prospective randomized comparative 1-year follow-up study. J Glaucoma. 2015;24(1):1-8. doi: 10.1097/IJG.0b013e318287abf3.

7. He M, Wang W, Zhang X, Huang W. Ologen implant versus mitomycin C for trabeculectomy: a systematic review and meta-analysis. PLoS One. 2014;9(1):e85782. doi: 10.1371/journal.pone.0085782.

8. Fujishima H, Shimazaki J, Shinozaki N, Tsubota K. Trabeculectomy with the use of amniotic membrane for uncontrollable glaucoma. Ophthalmic Surg Lasers. 1998;29(5):428-31.

9. Ji QS, Qi B, Liu L, Lao W, Yang ZH, Wang GF, et al. Comparison of trabeculectomy and trabeculectomy with amniotic membrane transplantation in the same patient with bilateral glaucoma. Int J Ophthalmol. 2013;6(4):448-51. doi: 10.3980/j.issn.2222-3959.2013.04.07.

10. Khairy HA, Elsawy MF. Trabeculectomy With Mitomycin-C Versus Trabeculectomy With Amniotic Membrane Transplant: A Medium-term Randomized, Controlled Trial. J Glaucoma. 2015;24(7):556-9. doi:

10.1097/IJG.0000000000000060.

11. Stavrakas P, Georgopoulos G, Milia M, Papaconstantinou D, Bafa M, Stavrakas E, et al. The use of amniotic membrane in trabeculectomy for the treatment of primary open-angle glaucoma: a prospective study. Clin Ophthalmol. 2012;6:205-12. doi: 10.2147/OPTH.S27187.

12. Sheha H, Kheirkhah A, Taha H. Amniotic membrane transplantation in trabeculectomy with mitomycin $\mathrm{C}$ for refractory glaucoma. J Glaucoma. 2008;17(4):303-7. doi: 10.1097/IJG.0b013e31815c3a47.

13. Yadava U, Jaisingh K, Dangda S, Thacker P, Singh K, Goel Y. Simultaneous use of amniotic membrane and Mitomycin C in trabeculectomy for primary glaucoma. Indian J Ophthalmol. 2017;65(11):1151-5. doi:

10.4103/ijo.IJO_447_17.

14. Mahdy RA, Nada WM, Almasalamy SM, Anany HA, Almasary AM. A freeze-dried (lyophilized) amniotic membrane transplantation with mitomycin $\mathrm{C}$ and trabeculectomy for pediatric glaucoma. Cutan Ocul Toxicol. 2010;29(3):164-70. doi: 10.3109/15569521003775005.

15. Dua HS, Gomes JA, King AJ, Maharajan VS. The amniotic membrane in ophthalmology. Surv Ophthalmol. 2004;49(1):51-77.

16. Zhong Y, Zhou Y, Wang K. [Effect of amniotic membrane on filtering bleb after trabeculectomy in rabbit eyes]. Yan Ke Xue Bao. 2000;16(2):73-6, 83.

17. Wang L, Liu X, Zhang P, Lin J. [An experimental trial of glaucoma filtering surgery with amniotic membrane]. Yan Ke Xue Bao. 2005;21(2):126-31.

18. Eliezer RN, Kasahara N, Caixeta-Umbelino C, Pinheiro RK, Mandia C, Jr., Malta RF. Use of amniotic membrane in trabeculectomy for the treatment of glaucoma: a pilot study. Arq Bras Oftalmol. 2006;69(3):309-12. 


\section{Figures}

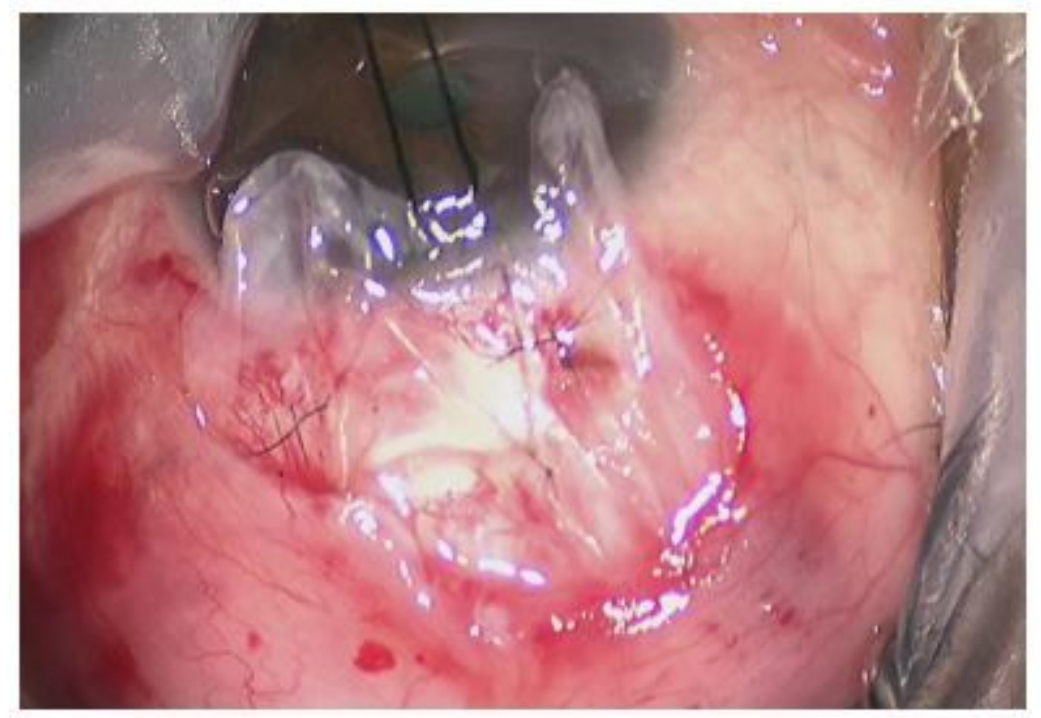

\section{Figure 1}

Amniotic membrane above the scleral flap (stromal surface facing down).

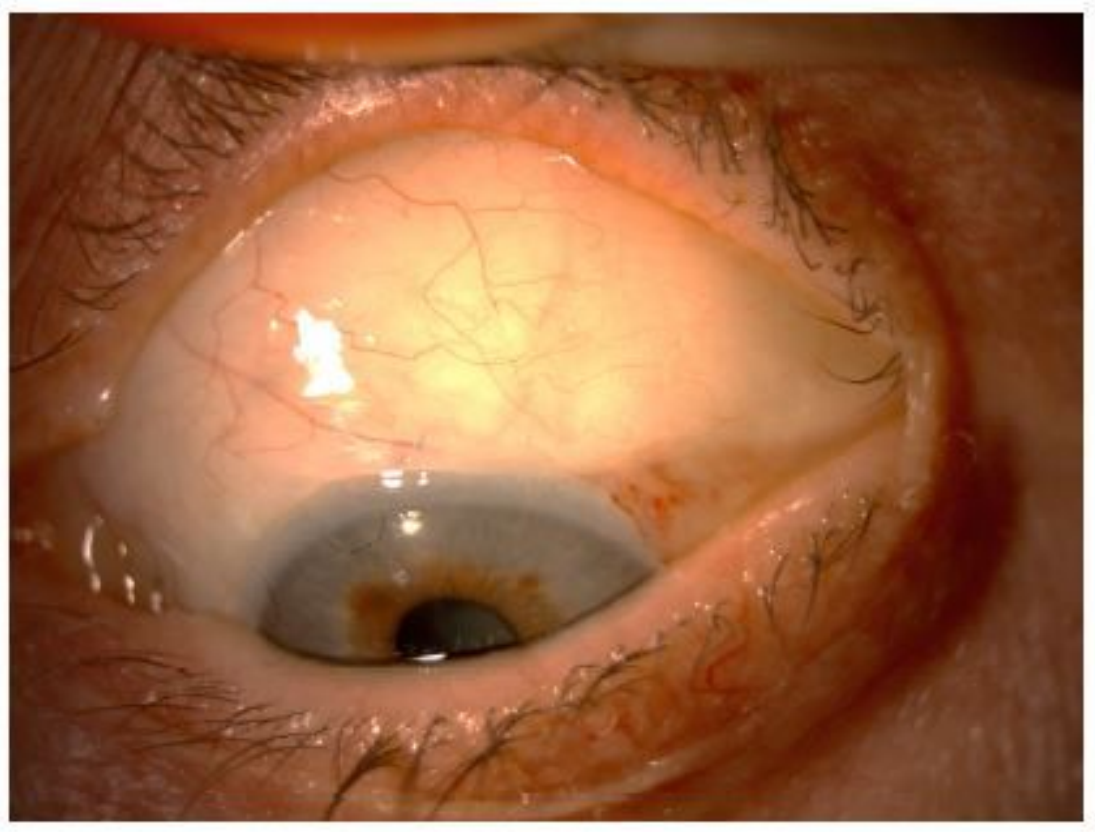

Figure 2

Large and diffuse filtration bleb registered in one of the eyes of the AMT group. 


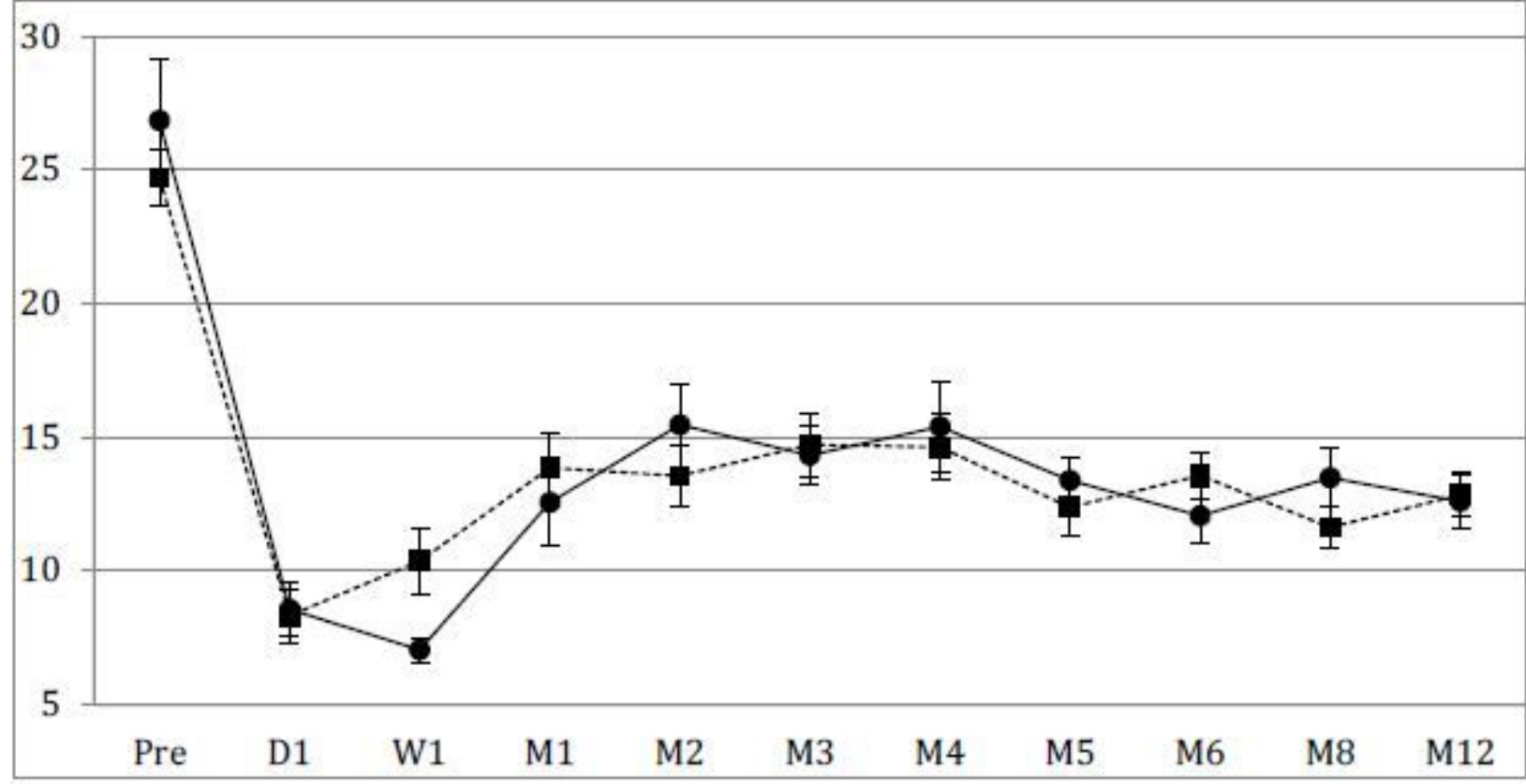

Figure 3

Preoperative and postoperative mean IOP during follow-up period. We present mean IOP values in each study group. The bars represent the standard error at each follow-up visit. Vertical axis- Mean IOP in each group; Horizontal axis- Preoperative and postoperative visits; Solid line- non-AMT group; Dashed lineAMT group.

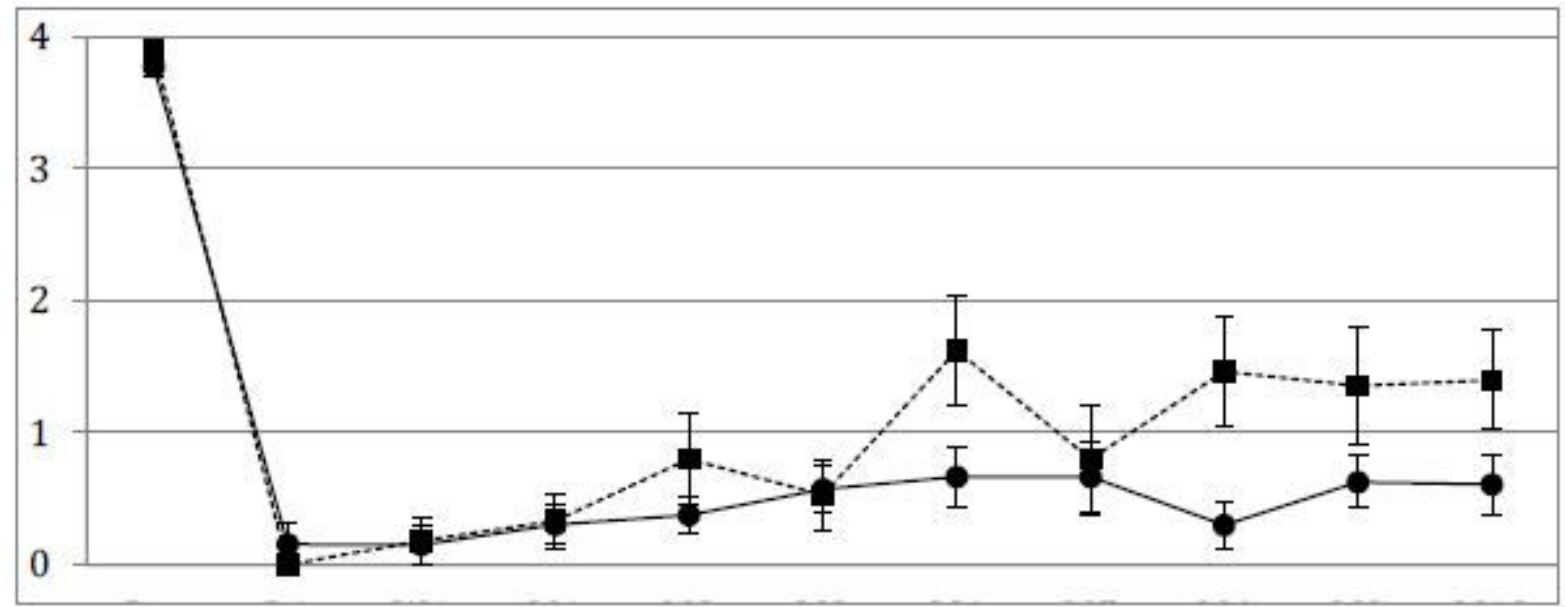

Figure 4

Preoperative and postoperative mean number of antihypertensive medications during follow-up period 